\title{
On the Submodularity of Multi-depot Traveling Salesman Games
}

\author{
Platz, Trine Tornøe
}

Document Version

Accepted author manuscript

Published in:

Discrete Applied Mathematics

DOI:

10.1016/j.dam.2018.11.029

Publication date:

2019

License

CC BY-NC-ND

Citation for published version (APA):

Platz, T. T. (2019). On the Submodularity of Multi-depot Traveling Salesman Games. Discrete Applied Mathematics, 255, 75-85. https://doi.org/10.1016/j.dam.2018.11.029

Link to publication in CBS Research Portal

\section{General rights}

Copyright and moral rights for the publications made accessible in the public portal are retained by the authors and/or other copyright owners and it is a condition of accessing publications that users recognise and abide by the legal requirements associated with these rights.

Take down policy

If you believe that this document breaches copyright please contact us (research.lib@cbs.dk) providing details, and we will remove access to the work immediately and investigate your claim. 


\title{
On the Submodularity of Multi-depot Traveling Salesman Games
}

\section{Trine Tornoe Platz}

\author{
Journal article (Accepted manuscript ${ }^{*}$ )
}

\section{Please cite this article as:}

Platz, T. T. (2019). On the Submodularity of Multi-depot Traveling Salesman Games. Discrete Applied Mathematics, 255, 75-85. 001: 10.1016/j.dam.2018.11.029

DOI: 10.1016/j.dam.2018.11.029

* This version of the article has been accepted for publication and undergone full peer review but has not been through the copyediting, typesetting, pagination and proofreading process, which may lead to differences between this version and the publisher's final version AKA Version of Record.

(1) 2019. This manuscript version is made available under the CC-BY-NC-ND 4.0 license http://creativecommons.org/licenses/by-nc-nd/4.0/ 


\title{
On the submodularity of multi-depot traveling salesman games
}

\author{
Trine Tornøe Platz ${ }^{\mathrm{a}}$ \\ ${ }^{a}$ Department of Economics, Copenhagen Business School, Porcelanshavne 16A, DK-2000 Frederiksberg, Denmark.
}

\begin{abstract}
The Steiner traveling salesman problem is the problem of finding a minimum cost tour for a salesman that must visit a set of locations while traveling along costly streets before returning to his starting point at the depot. A solution to the problem is a minimum cost Steiner tour that both starts and ends at the depot and visits all the required locations. If different players are associated with the locations to be visited, the problem induces a cooperative traveling salesman (TS) game that poses the question of how to allocate the total cost of the tour between the different players involved. This cost allocation problem can be tackled using tools and solutions from cooperative game theory.

The purpose of this paper is to generalize the notion of a traveling salesman game to allow for multiple depots in the underlying traveling salesman problem (TSP) and to analyse the submodularity of such multi-depot TS games. A multi-depot TSP can be represented by a connected (di)graph in which a fixed set of vertices are denoted depots, and a non-negative weight function is defined on the edges of the graph. The submodularity of multi-depot TS games is analysed by characterizing graphs and digraphs that induce submodular multi-depot TS games for any choice of depots and for at least one choice of the depots, respectively.
\end{abstract}

Keywords: Steiner traveling salesman problem, cooperative game, submodularity 2000 MSC: $91 \mathrm{~A} 12$

\section{Introduction}

The Steiner traveling salesman problem models the situation in which a salesman affiliated with a fixed depot (warehouse) has to visit a number of locations before returning to the depot. There is a cost associated with traveling along the roads that connect locations, and a solution to the problem is a minimum cost Steiner tour that visits the required locations while starting and ending at the depot. Since a Steiner tour allows multiple visits to the same vertices and also allows the set of vertices to be visited to be only a subset of the vertices of the graph, this variant of the traveling salesman problem can be considered more suitable for some applications, including applications on real world road networks, e.g. Letchford et al. 9]. The traveling salesman problem has been extended in a number of directions, for example by allowing for multiple salesmen, multiple warehouses, and by adding restrictions on visiting times and capacity.

A traveling salesman problem (TSP) can be represented by a (di)graph in which a fixed vertex is denoted the depot, and a weight function is defined on the edges (arcs). If the vertices to be visited are associated with different players, the TSP induces a cooperative cost allocation game denoted a traveling salesman game [12, 14 in which the cost of a coalition of players is the cost of a minimum weight tour that visits all players in the coalition. Once the cooperative game is defined, tools and solution concepts from cooperative game theory can be applied to analyze and solve the cost allocation problem.

The purpose of this paper is to extend the notion of a traveling salesman game to allow for multiple depots in the underlying TSP and to consider the submodularity of such multi-depot TS games. The

Email address: ttp.eco@cbs.dk (Trine Tornøe Platz) 
multi-depot setup considered here may be interpreted as a situation in which several depots exist, each with their own salesman/vehicle of unlimited capacity, such that an optimal tour may be a collection of subtours each originating from a different depot and returning to it's point of origin.

The focus on submodularity is motivated by the desirable properties of submodular games. For one thing, submodular games are totally balanced, implying that the core of every subgame is non-empty. The Shapley value is in the core of a submodular game, and it is the barycenter of the core, Shapley 13. Furthermore, several solution concepts coincide (the nucleolus and the kernel, the bargaining set and the core, Maschler et al. [10]), and others can be more easily computed for this class of games than is generally the case.

In the following, a multi-depot TSP with $k$ depots will be denoted a $k$-depot TSP or simply, a $k$-depot TS problem. Furthermore, a graph $G$ is said to be globally (locally) $k$-TS submodular if the game induced by a $k$-depot TS problem on $G$ is submodular for every (some) location of the $k$ depots and for every weight function. This paper characterizes the classes of globally and locally $k$-TS submodular undirected graphs and also provide some results on the charaterization of globally and locally $k$-TS submodular digraphs. For the undirected case, it is shown that a graph $G$ is globally $k$-TS submodular for $k \in\{2, \ldots,|V(G)|-3\}$ if and only if $G$ does not contain a path of 5 vertices or more. Furthermore, a graph $G$ is locally $k$-TS submodular if a set of $k$ depots can be allocated such that a specific pattern is avoided, and if $G$ at the same time fulfills a cut condition for all connected components in a specific subgraph of $\mathrm{G}$.

Likewise, directed graphs are globally $k$-TS submodular only if they do not contain specific forbidden structures, and it is also shown that the class of locally $k$-TS submodular graphs is a strict superset of the globally $k$-TS submodular graphs.

For the standard case with just one depot in the underlying TSP, Herer and Penn 8 provided a characterization of the undirected graphs that induce submodular traveling salesman games. This result was extended to the directed case in Granot et al. [4]. The games analyzed in these papers, can be seen as special cases of the multi-depot TS game, for which $k=1$. In a related string of research, Granot et al. 6] characterized for the case of Chinese postman (CP) games both CP-balanced and CP-submodular graphs. In Granot and Hamers [5, the authors distinguished between global and local requirements and characterized the class of locally CP-submodular graphs as well as locally TS-submodular graphs. The approach of analyzing both global and local requirements is followed in the present paper. Another related paper is Platz and Hamers 11 that introduce multi-depot Chinese postman games and characterize the classes of (globally and locally) $k$-CP balanced and $k$-CP submodular graphs. The modelling of the multi-depot TS game in the present paper follows a similar approach, but due to the different combinatorial nature of the two problems, the results and proofs differ.

The analysis of traveling salesman games (and Chinese postman games) is part of a larger literature that analyzes the properties of cooperative games that arise from underlying optimization problems. A few other examples of such classes of games are minimum cost spanning tree games [7, sequencing games [2], and minimum colouring games [3. An overview can be found in Curiel [1].

The paper is structured as follows. Section 2 presents some useful terms and notation. In section 3 , multi-depot traveling salesman games are introduced. Results are presented in sections 4 and 5 for undirected and directed graphs respectively.

\section{Preliminaries}

From cooperative game theory, we recall the following definitions: In a cooperative (cost) game $(N, c), N=\{1, \ldots, n\}$ denotes the finite playerset, and $c: 2^{N} \rightarrow \mathbb{R}$ is a function that assigns to every coalition $S \subseteq N$ a cost $c(S)$, with $c(\emptyset)=0$. Let $x \in \mathbb{R}^{N}$ be an allocation of $c(N)$ between the players. A game $(N, c)$ is monotonic if $c(S) \leq c(T)$ for all $S \subset T \subseteq N$, and it is subadditive if $c(S \cup T) \leq c(S)+c(T)$ for all $S, T \subseteq N$ with $S \cap T=\emptyset$. The core of a game $(N, c)$ is given by

$$
\operatorname{core}(N, c)=\left\{x \in \mathbb{R}^{N} \mid \sum_{i \in N} x_{i}=c(N), \sum_{i \in S} x_{i} \leq c(S) \text { for all } S \subseteq N\right\} .
$$


The game $(N, c)$ is said to be submodular if

$$
c(T \cup\{i\})-c(T) \leq c(S \cup\{i\})-c(S)
$$

for all $i \in N$ and all $S \subset T \subseteq N \backslash\{i\}$.

Next, we recall some notions from graph theory. An undirected (directed) graph $G=(V(G), E(G))$ consists of a non-empty, finite set of vertices $V(G)$ and a set of pairs of vertices $E(G)$ called edges (arcs). An edge $\{a, b\}$ in an undirected graph or an arc $(a, b)$ in a directed graph joins the vertices $a, b$ and is said to be incident to $a$ and $b$. The vertices $a$ and $b$ are adjacent. The degree of a vertex $a$ is equal to the number of edges incident to $a$. An $\operatorname{arc}(a, b)$ is directed from $a$ to $b$ and can only be traversed in this direction.

A walk (respectively, directed walk), $w$, in a graph $G$ is a sequence of vertices and edges (respectively, arcs) of the form: $v_{0}, e_{1}, v_{1}, \ldots, v_{m-1}, e_{m}, v_{m}$, where $v_{0}, \ldots, v_{m} \in V(G), e_{1}, \ldots, e_{m} \in E(G)$, and $m \geq 0$ such that $e_{j}=\left\{v_{i-1}, v_{j}\right\}$ (respectively, $e_{j}=\left(v_{i-1}, v_{j}\right)$ ) for all $i \in\{1, \ldots m\}$. If $v_{0}=v_{m}$, the walk is said to be closed. A closed walk may be empty, $w=\left\{v_{0}\right\}$. A (directed) path is a (directed) walk in which no edge (arc) or vertex is visited more than once, except $v_{0}$ in the case of $v_{0}=v_{m}$. The length of a (directed) path is equal to the number, $m$, of edges (arcs) traversed. If there exists an undirected (directed) path between any two vertices in a (directed) graph $G$, then $G$ is a (strongly) connected graph. A closed (directed) walk in which no edge is visited more than once will be denoted a (directed) circuit, whereas a closed (directed) path, i.e., a (directed) walk in which no edge (arc) or vertex is visited more than once, is denoted a (directed) cycle. A subdivision of a graph $G$ is the graph $G^{\prime}$ that arises by (repeatedly) replacing an edge (arc) in $G$ with a (directed) path of length two.

Let $G$ be a graph, and let $v_{s}$ and $v_{t}$ be two vertices in $G$. Then an $\mathrm{s}-\mathrm{t}$ vertex cutset is a set of vertices such that removing these vertices along with the edge $\left\{v_{s}, v_{t}\right\}$, if it exists, results in a disconnected graph in which $v_{s}$ and $v_{t}$ do not belong to the same component. A minimal $\mathrm{s}-\mathrm{t}$ vertex cutset, $K$, is an s - t vertex cutset such that no proper subset of $K$ is an $\mathrm{s}$ - t vertex cutset.

Finally, an outerplanar graph is a graph that can be embedded in the plane such that no edges cross, and such that all vertices of the graph lie on the boundary of the outer face of the embedding.

\section{Multi-depot traveling salesman games}

Let $G=((V(G), E(G))$ be a (strongly) connected (di)graph, and let $Q \subset V(G)$ be a fixed subset of the vertices of $G$. An element of $Q$ is called a depot. A multi-depot traveling salesman problem is given by a tuple $\Gamma=\left(V^{-}(G),(G, Q), t\right)$ in which $V^{-}(G)=V(G) \backslash Q$ denotes the set of vertices in $G$ that are not associated with depots, and $t: E(G) \rightarrow[0: \infty)$ is a weight function defined on the edges (arcs) of $G$.

Let $w=\left(v_{0}, e_{1}, v_{1}, \ldots, e_{m}, v_{m}\right)$ denote a walk in $G$. The cost of $w$ equals the sum of the weights of the edges traversed, such that $\operatorname{cost}(w)=\sum_{i=1}^{m} t\left(e_{i}\right)$. A closed walk that starts and ends at a vertex $v \in Q$ is denoted $w_{v}$. A closed walk $w_{v}=\{v\}$ is said to be empty, and the cost of an empty walk is 0 .

Let $G$ be a (strongly) connected (di)graph, and let $\Gamma=\left(V^{-}(G),(G, Q), t\right)$ be a multi-depot TSP defined on $G$. Next, let $S \subseteq V^{-}(G)$ be a subset of the non-depot vertices of $G$. Then for a given $Q=\left\{v_{1}, \ldots, v_{k}\right\}$, an $S$-Steiner tour $d(S)$ is a collection of closed walks $d(S)=\left\{w_{v_{1}}, \ldots, w_{v_{k}}\right\}$ (some of which may be empty) such that every vertex in $S$ is visited. The set of all $S$-Steiner tours is denoted $D(S)$.

The following example illustrates the notion of an $S$-Steiner tour.

Example 3.1. Consider Figure 1 in which depots are located at vertices $v_{0}$ and $v_{2}$, whereas $v_{1}$ and $v_{3}$ are associated with players. There are then at least two possible $S$-Steiner tours for $S=\left\{v_{1}, v_{3}\right\}$. One is the tour $v_{2}, e_{2}, v_{1}, e_{2}, v_{2}, e_{3}, v_{3}, e_{3}, v_{2}$, and the other is the tour consisting of the two subtours $v_{0}, e_{1}, v_{1}, e_{1}, v_{0}$ and $v_{2}, e_{3}, v_{3}, e_{3}, v_{2}$. 
Figure 1: $S$-Steiner tours in a multi-depot setting

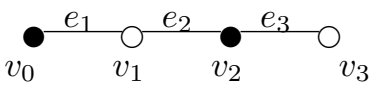

When depots are located at the vertices of $Q$, the cost of an $S$-Steiner tour $d(S)=\left\{w_{v_{1}}, \ldots, w_{v_{k}}\right\}$ is equal to:

$$
C_{Q}(d(S))=\sum_{i=1}^{k} \operatorname{cost}\left(w_{v_{i}}\right)
$$

Now, the multi-depot TS game induced by a multi-depot TSP can be defined as follows. Let $G=(V(G), E(G))$ be a (strongly) connected (directed) graph, and let $\Gamma=\left(V^{-}(G),(G, Q), t\right)$ be a multi-depot TSP defined on $G$. Then $\left(N, c_{Q}\right)$ is the induced multi-depot TS game in which $N=V^{-}(G)$ is the set of players, and $c_{Q}(S)$ is, for any $S \subseteq N$, the cost of a minimum weight $S$-Steiner tour, when the depots are located at the vertices of $Q$. That is:

$$
c_{Q}(S)=\min _{d(S) \in D(S)} C_{Q}(d(S)) .
$$

Note that since $N=V^{-}(G)=V(G) \backslash Q$, different sets of depots imply different sets of players, and in particular, a greater number of depots implies fewer players. An illustration of two 2-depot TS problems and their induced games are given in the example below.

Example 3.2. The graph in Figure 2 illustrates two different 2-depot TS problems defined on the same graph. For the problem on the left, $Q=\left\{v_{0}, v_{4}\right\}$ while $Q=\left\{v_{0}, v_{3}\right\}$ for the problem on the right. For both TSPs assume that $t\left(\left\{v_{1}, v_{4}\right\}\right)=10$ while $t(e)=1$ for all other edges in the graph. The two induced 2-depot TS games $\left(\{1,2,3\}, c_{\left\{v_{0}, v_{4}\right\}}\right)$ and $\left(\{1,2,4\}, c_{\left\{v_{0}, v_{3}\right\}}\right)$ are shown in the table below. Note that as the location of the depots change, so does the player set. Furthermore, with the current choice of weight function, we see that $c_{\left\{v_{0}, v_{4}\right\}}$ is not submodular since $c_{\left\{v_{0}, v_{4}\right\}}\left(v_{1}, v_{2}, v_{3}\right)-c_{\left\{v_{0}, v_{4}\right\}}\left(v_{2}, v_{3}\right)=$ $2>0=c_{\left\{v_{0}, v_{4}\right\}}\left(v_{1}, v_{2}\right)-c_{\left\{v_{0}, v_{4}\right\}}\left(v_{2}\right)$, whereas it is easy to verify that $c_{\left\{v_{0}, v_{3}\right\}}$ is (sub)modular.

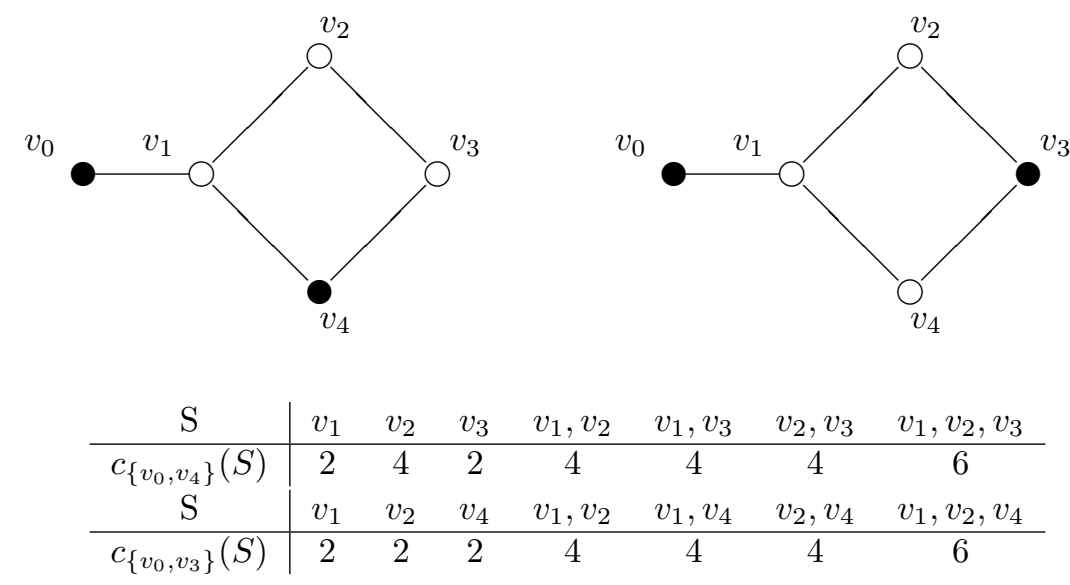

Figure 2: Two 2-depot TS problems and their induced 2-depot TS games

Whereas not every $k$-depot TS game is submodular, it is straightforward to verify that for any multi-depot TS game $\left(N, c_{Q}\right)$ induced by a multi-depot TSP, $\Gamma=\left(V^{-}(G),(G, Q), t\right)$, it holds that $c_{Q}(S) \leq c_{Q}(T)$ for all $S \subset T \subseteq N$ (the game is monotonic), and $c_{Q}(S \cup T) \leq c_{Q}(S)+c_{Q}(T)$ for all $S, T \subseteq N$ with $S \cap T=\emptyset$ (the game is subadditive). 
We now turn to the analysis of multi-depot traveling salesman games. We will consider both undirected and directed graphs, and characterize classes of graphs that give rise to submodular multidepot TS games. Since any subadditive two-player game is submodular, only games of at least three players are considered. Therefore, $|V(G)| \geq k+3$ for all $k$-depot TS problems throughout the paper.

\section{4. $k$-TS submodular undirected graphs}

\subsection{Globally k-TS submodular graphs}

Before proceeding to the characterization of graphs, a definition and a few useful observations are stated.

Let $P_{m}$ denote a path with $m$ vertices. Let $P_{m+1}^{F}$ denote the structure illustrated in Figure 3 in which depots are located only at the endpoints of a path with $m+1$ vertices and $m \geq 4$.

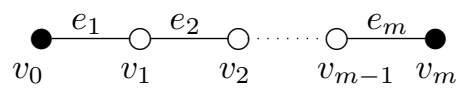

Figure 3: The $P_{m+1}^{F}$ structure on a path of length $m \geq 4$

Definition 4.1. Let $\Gamma=\left(V^{-}(G),(G, Q), t\right)$ be a multi-depot TS problem defined on a connected graph $G$. Then $G$ is $P_{m+1}^{F}$-free with respect to $Q$, if no path $v_{0}, e_{1}, v_{1}, \ldots, e_{m}, v_{m}$ exists in $G$ such that $v_{0}, v_{m} \in Q, v_{1}, \ldots, v_{m-1} \in V^{-}(G)$ and $m \geq 4$.

It turns out that the presence of a $P_{m+1}^{F}$ structure with $m \geq 4$ is incompatible with submodularity of the induced multi-depot TS game, as will be clear in what follows.

We are now ready to characterize globally $k$-TS submodular graphs for $1<k<|V(G)|-2$. Recall that for $k=1$, a characterization of globally 1-depot TS-submodular graphs was given in Herer and Penn (1995), and for $k \in\{|V(G)|-2,|V(G)|-1\}$, all connected graphs are globally $k$-TS submodular, since there are at most two players in the induced game.

Theorem 4.1. Let $G=(V(G), E(G))$ be a connected, undirected graph, and let $k \in\{2, \ldots,|V(G)|-3\}$. Then $G$ is globally $k$-TS submodular if and only if $G$ contains no path of length four.

Proof. Let $\Gamma=\left(V^{-}(G),(G, Q), t\right)$ be a $k$-depot TS problem on $G$, and let $\left(N, c_{Q}\right)$ be the induced $k$-depot TS game.

First, for the 'only if' part assume that $G$ contains a path of length four. Then there exists a pair $\left(v_{i}, v_{i+4}\right)$ of distinct vertices in $G$ such that there is a path $P:=v_{i}, e_{i+1}, v_{i+1}, \ldots, e_{i+4}, v_{i+4}$, from $v_{i}$ to $v_{i+4}$ visiting four edges. However, since $|Q|=k \leq|V(G)|-3$, we can then choose $Q$ such that $v_{i}, v_{i+4} \in Q$, and $v_{i+1}, \ldots, v_{i+3} \in V^{-}(G)$, implying that $G$ is not $P_{m+1}^{F}$-free with respect to $Q$. Next, let $t\left(e_{j}\right)=1$ for $j \in\{i+1, i+2, i+3, i+4\}$, and let $t(e)=10$ for all other edges in $G$. Then $c_{Q}\left(v_{i+1}, v_{i+2}, v_{i+3}\right)-c_{Q}\left(v_{i+1}, v_{i+2}\right)=6-4=2$ whereas $c_{Q}\left(v_{i+2}, v_{i+3}\right)-c_{Q}\left(v_{i+2}\right)=4-4=0$, thus violating (2.1), and $c_{Q}$ is not submodular. Therefore, since the induced game is not submodular for all weight functions, $G$ is not globally $k$-TS submodular.

Now, consider the 'if' part and assume that $G$ contains no path of length four. Since $|V(G)| \geq k+3$, $G$ has at least 5 vertices. Next, note that the only graphs with five vertices or more that do not contain a path of length four are: star graphs with at least four pendant vertices, graphs obtained by joining two star graphs by adding an edge between the two center vertices (a double star), and graphs obtained from a star graph by adding a single edge between two pendant vertices. The latter two types of graphs are illustrated in Figure 4. In all of these three cases, it holds that if $S=\emptyset$, then $c_{Q}(S \cup\{v\})-c_{Q}(S)=c(v)$ for all $v \in N$, and it follows from subadditivity of $c$ that (2.1) holds. In the remaining part of the proof, we may therefore assume that $S \neq \emptyset$. The three types of graphs will be considered separately. 

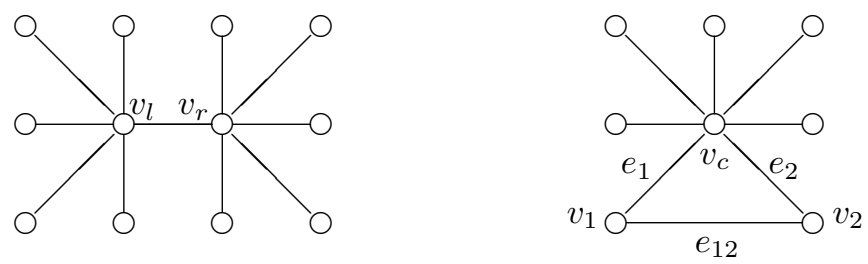

Figure 4: Globally $k$-TS submodular graph types

First, assume that $G$ is a star graph, and let the single vertex with degree larger than one be denoted $v_{c}$. For any $v \in V(G) \backslash\left\{v_{c}\right\}$, let $e_{v}$ denote the edge connecting $v$ to the center vertex $v_{c}$. Now, if $S \neq \emptyset$, then $v_{c} \in d(S)$, irrespective of the location of depots in $G$. Then, for all $v \in N$ and all $S \subset T \subseteq N \backslash\{v\}$, we have

$$
c_{Q}(T \cup\{v\})-c_{Q}(T)=c_{Q}(S \cup\{v\})-c_{Q}(S)=\left\{\begin{array}{cl}
0 & \text { if } v=v_{c} \\
2 t\left(e_{v}\right) & \text { otherwise },
\end{array}\right.
$$

and (2.1) holds.

Second, let $G$ be a double star graph. Let the two vertices with degree greater than 1 be denoted $v_{l}$ (left-center) and $v_{r}$ (right-center) respectively, as in Figure 4. For $v_{i} \in V(G) \backslash\left\{v_{l}, v_{r}\right\}$ let $e_{i}$ denote the edge incident to $v_{i}$. Assume that $S \neq \emptyset$ and note that when coalition $S$ is non-empty, either $v_{l}$ or $v_{r}$ must belong to $d(S)$. Assume that $v_{l} \in d(S)$, and let $e^{*}$ denote the cheapest edge connecting $v_{r}$ to a vertex in $Q \cup\left\{v_{l}\right\}$ (symmetric arguments can be applied if $v_{r} \in d(S)$ ). Let $v_{i} \in V^{-}(G)$ and consider three separate cases:

Case 1. $v_{i} \in\left\{v_{l}, v_{r}\right\}$ : If $v_{i}=v_{l}$ then $c_{Q}\left(T \cup\left\{v_{i}\right\}\right)-c_{Q}(T)=c_{Q}\left(S \cup\left\{v_{i}\right\}\right)-c_{Q}(S)=0$ since $v_{l} \in d(S)$. If $v_{i}=v_{r}$ then $c_{Q}\left(T \cup\left\{v_{i}\right\}\right)-c_{Q}(T)=c_{Q}\left(S \cup\left\{v_{i}\right\}\right)-c_{Q}(S)=0$, if $v_{r} \in d(S)$, and $c_{Q}\left(T \cup\left\{v_{i}\right\}\right)-c_{Q}(T) \leq c_{Q}\left(S \cup\left\{v_{i}\right\}\right)-c_{Q}(S)=2 t\left(e^{*}\right)$ otherwise.

Case 2. $v_{i} \in V^{-}(G) \backslash\left\{v_{l}, v_{r}\right\}$ and $v_{i}$ is adjacent to $v_{l}$ : then $c_{Q}\left(T \cup\left\{v_{i}\right\}\right)-c_{Q}(T)=c_{Q}\left(S \cup\left\{v_{i}\right\}\right)-c_{Q}(S)=$ $2 t\left(e_{i}\right)$.

Case 3. $v_{i} \in V^{-}(G) \backslash\left\{v_{l}, v_{r}\right\}$ and $v$ is adjacent to $v_{r}$ : then $c_{Q}\left(T \cup\left\{v_{i}\right\}\right)-c_{Q}(T)=c_{Q}\left(S \cup\left\{v_{i}\right\}\right)-c_{Q}(S)=$ $2 t\left(e_{i}\right)$ if $v_{r} \in\{Q \cup d(S)\}$, and $c_{Q}\left(T \cup\left\{v_{i}\right\}\right)-c_{Q}(T) \leq 2 t\left(e_{i}\right)+2 t\left(e^{*}\right)=c_{Q}\left(S \cup\left\{v_{i}\right\}\right)-c_{Q}(S)$ otherwise.

It follows that (2.1) holds in all three cases, and the induced game is submodular for every $Q \subset V(G)$ and all weight functions.

Lastly, consider the graph in the right panel of Figure 4. Let the single vertex of degree larger than 2 be denoted $v_{c}$ and refer to it as the center vertex. Let the two 2-degree vertices be denoted $v_{1}$ and $v_{2}$ respectively, and let $e_{12}$ denote the edge incident to both $v_{1}$ and $v_{2}$. Furthermore, for any $v_{i} \in V(G) \backslash\left\{v_{c}\right\}$, let $e_{i}$ denote the edge connecting $v_{i}$ to $v_{c}$, and let $e^{*}$ denote the minimum weight edge connecting $v_{c}$ to a vertex in $Q \cup\left\{v_{1}, v_{2}\right\}$. Assume that $S \neq \emptyset$, and consider two cases separately.

Case 1. $v_{c} \notin d(S)$ : then since $S \neq \emptyset$, and the center vertex is not visited, it must be that one of the two vertices $\left\{v_{1}, v_{2}\right\}$ is in $S$ while the other is in $Q$, and futhermore, that $\left\{v_{1}, v_{2}\right\}$ are the only vertices visited by $d(S)$. Thus, either $S=\left\{v_{1}\right\}$ and $v_{2} \in Q$, or $S=\left\{v_{2}\right\}$ and $v_{1} \in Q$. Either way, it must be that $v_{c} \in d(T)$ for all $S \subset T$. Therefore, if $v=v_{c}$, we get $c_{Q}(T \cup\{v\})-c_{Q}(T)=0<2 t\left(e^{*}\right)=$ $c_{Q}(S \cup\{v\})-c_{Q}(S)$. For $v=v_{i} \in V^{-}(G) \backslash\left\{v_{c}, v_{1}, v_{2}\right\}$, we have $c_{Q}\left(T \cup\left\{v_{i}\right\}\right)-c_{Q}(T)=2 t\left(e_{i}\right)$, while $c_{Q}\left(S \cup\left\{v_{i}\right\}\right)-c_{Q}(S)=2 t\left(e_{i}\right)+2 t\left(e^{*}\right)$ when $v_{c} \notin Q$, and $c_{Q}\left(S \cup\left\{v_{i}\right\}\right)-c_{Q}(S)=2 t\left(e_{i}\right)$ when $v_{c} \in Q$.

Case 2. $v_{c} \in d(S)$ : then it is trivial that $c_{Q}\left(T \cup\left\{v_{c}\right\}\right)-c_{Q}(T)=c_{Q}\left(S \cup\left\{v_{c}\right\}\right)-c_{Q}(S)=0$, and that $c_{Q}\left(T \cup\left\{v_{i}\right\}\right)-c_{Q}(T)=c_{Q}\left(S \cup\left\{v_{i}\right\}\right)-c_{Q}(S)=2 t\left(e_{i}\right)$ for $v_{i} \in V^{-}(G) \backslash\left\{v_{c}, v_{1}, v_{2}\right\}$. Therefore, consider the case of $v=v_{1}$ (symmetric arguments can be applied to the case of $v=v_{2}$ ). Then either a) $v_{1} \in d(S)$ which, again, trivially implies $c_{Q}\left(T \cup\left\{v_{1}\right\}\right)-c_{Q}(T)=c_{Q}\left(S \cup\left\{v_{1}\right\}\right)-c_{Q}(S)=0$, or b) $v_{1} \notin$ $d(S), v_{2} \in d(S)$, in which case $c_{Q}\left(T \cup\left\{v_{1}\right\}\right)-c_{Q}(T)=c_{Q}\left(S \cup\left\{v_{1}\right\}\right)-c_{Q}(S)=\min \left\{2 t\left(e_{1}\right), 2 t\left(e_{12}\right)\right\}$, or c) 
$v_{1}, v_{2} \notin d(S)$, implying that $c_{Q}\left(S \cup\left\{v_{1}\right\}\right)-c_{Q}(S)=\min \left\{2 t\left(e_{1}\right), 2 t\left(e_{2}\right)+2 t\left(e_{12}\right)\right\} \geq c_{Q}\left(T \cup\left\{v_{i}\right\}\right)-c_{Q}(T)$, where the inequality follows, since $v_{c} \in d(S) \Rightarrow v_{c} \in d(T)$. Thus, 2.1 holds.

Now, consider the following definition:

Definition 4.2. Let $G$ be a connected, undirected graph. Then $G$ satisfies the cut condition if there is no vertex-pair $v_{s}, v_{t} \in V(G)$ such that there exists a minimal s-t vertex cutset of cardinality greater than 2 .

From Herer and Penn (1995) it follows that an undirected graph is globally 1-TS submodular if and only if it satisfies the cut condition. Furthermore, from the proof of Theorem 4.1 it is easy to verify that all graphs that are globally $k$-TS submodular for $k>1$ satisfy the cut condition. Hence, the class of globally $k$-TS submodular graphs is a subset of the globally 1-TS submodular graphs.

\subsection{Locally $k$-TS submodular graphs}

When modelling situations in which we can freely choose the location of depots, requiring the induced game to be submodular for all possible locations of depots seems too strong a restriction, and it may be more relevant to simply ask whether there exists at least one location of depots that induces a submodular game for every possible weight function. Therefore, it is also relevant to characterize the class of locally $k$-TS submodular graphs. Recall that a graph $G=(V(G), E(G))$ is said to be locally $k$-TS submodular if there exists $Q \subset V(G)$, with $|Q|=k$, such that the induced $k$-TS game $\left(N, c_{Q}\right)$ is submodular for all weight functions.

In Granot and Hamers [5, it was shown that the class of locally 1-depot TS submodular graphs is equivalent to the class of globally 1-depot TS submodular graphs. A similar result does not hold for $k$-TS submodular graphs when $k>1$. For $k>1$, the class of locally $k$-TS submodular graphs is a strict superset of the class of globally $k$-TS submodular graphs. The class of locally $k$-TS submodular graphs can be characterized as follows.

Theorem 4.2. Let $G=(V(G), E(G))$ be a connected graph, and let $Q \subset V(G)$ be a subset of the vertices with cardinality $k \in\{2, \ldots,|V(G)|-3\}$. Let $G_{Q}$ denote the subgraph induced by all paths between vertices of $Q$. Then $G$ is locally $k$-TS submodular if and only if there exists $Q \subset V(G)$ such that $G$ is $P_{m+1}^{F}$-free with respect to $Q$ for $m \geq 4$, and such that all connected components of $G \backslash E\left(G_{Q}\right)$ satisfy the cut condition.

Proof. Let $\Gamma=\left(V^{-}(G),(G, Q), t\right)$, and let $\left(N, c_{Q}\right)$ be the induced $k$-depot TS game. Consider first the 'if part' and assume that there exists $Q \subset V(G)$ such that $G$ is $P_{m+1}^{F}$-free with respect to $Q$ for $m \geq 4$. This implies that a path between vertices in $Q$ can visit at most two player vertices in a row, and that every player vertex in $V\left(G_{Q}\right)$ is adjacent to at least one depot and at most one other player vertex within $G_{Q}$, as illustrated in Figure 5 A player vertex $v_{j} \in V\left(G_{Q}\right)$ can also be adjacent to players in $G \backslash V\left(G_{Q}\right)$. When this is the case, let $G_{j}$ denote the connected component in $G \backslash E\left(G_{Q}\right)$ that contains $v_{j}$ and note that $v_{j}$ must be visited on any tour visiting players in $G_{j}{ }^{1}$ Therefore, it holds for all $S \subset T \subseteq N \backslash\left\{v_{i}\right\}$ that if $V\left(G_{j}\right) \cap S \neq \emptyset$, then $v_{j}$ is visited by both $d(S)$ and $d(T)$. It remains to be shown that (2.1) holds for all players $v_{i} \in N$ and all $S \subset T \subseteq N \backslash\left\{v_{i}\right\}$. We distinguish between two cases.

Case 1. $v_{i} \in V\left(G_{Q}\right)$. For any vertex $v_{l} \in V\left(G_{Q}\right) \cap N$, let $e_{l}$ denote the minimum weight edge connecting $v_{l}$ to a depot. We start by showing that if $v_{i} \in d(S)$, then $v_{i} \in d(T)$ as well. To see this, note that if $v_{i} \in d(S)$, then it must be that either a) $V\left(G_{i}\right) \cap S \neq \emptyset$, or b) that $v_{i}$ is adjacent to a player vertex $v_{j}$, $V\left(G_{j}\right) \cap S \neq \emptyset$, and $t\left(e_{i}\right)+t\left(e_{i j}\right) \leq t\left(e_{j}\right)$, where $e_{i j}$ denotes the edge $\left\{v_{i}, v_{j}\right\}$. However, from a), it follows that $V\left(G_{i}\right) \cap T \neq \emptyset$, and hence that $v_{i} \in d(T)$, and likewise, if b) holds, it follows that $V\left(G_{j}\right) \cap T \neq \emptyset$

\footnotetext{
${ }^{1}$ In case $v_{j}$ is not adjacent to any players in $G \backslash V\left(G_{Q}\right)$, we simply have $G_{j}=\left\{v_{j}\right\}$.
} 


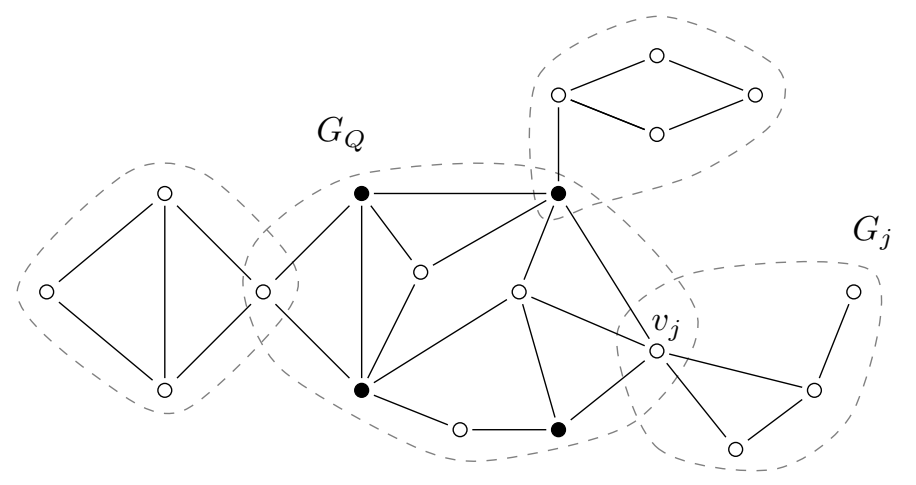

Figure 5: $G$ locally $k$-TS submodular

(along with $t\left(e_{i}\right)+t\left(e_{i j}\right) \leq t\left(e_{j}\right)$ ), implying that $v_{i} \in d(T)$. Therefore, $v_{i} \in d(S) \Rightarrow v_{i} \in d(T)$. In general, we know that if $v_{i} \in d(T)$, then $c_{Q}\left(T \cup\left\{v_{i}\right\}\right)-c_{Q}(T)=0 \leq c_{Q}\left(S \cup\left\{v_{i}\right\}\right)-c_{Q}(S)$, and condition (2.1) holds, so assume on the contrary that $v_{i} \notin d(S), v_{i} \notin d(T)$. First, if $v_{i}$ is not adjacent to any player vertex, it follows immediately that $c_{Q}\left(T \cup\left\{v_{i}\right\}\right)-c_{Q}(T)=2 t\left(e_{i}\right)=c_{Q}\left(S \cup\left\{v_{i}\right\}\right)-c_{Q}(S)$. Next, assume that $v_{i}$ is adjacent to player vertex $v_{j}$. Then if $v_{j} \in d(S)$, it follows that $v_{j} \in d(T)$ and therefore, $c_{Q}\left(S \cup\left\{v_{i}\right\}\right)-c_{Q}(S)=\min \left\{2 t\left(e_{i}\right), 2 t\left(e_{i j}\right)\right\}=c_{Q}\left(T \cup\left\{v_{i}\right\}\right)-c_{Q}(T)$. On the other hand, if $v_{j} \notin d(S)$, then $c_{Q}\left(S \cup\left\{v_{i}\right\}\right)-c_{Q}(S)=\min \left\{2 t\left(e_{i}\right), 2 t\left(e_{i j}\right)+2 t\left(e_{j}\right)\right\} \geq c_{Q}\left(T \cup\left\{v_{i}\right\}\right)-c_{Q}(T)$. In all three cases, (2.1) holds.

Case 2. $v_{i} \notin V\left(G_{Q}\right)$. Then there exists a vertex $v_{0} \in V\left(G_{Q}\right)$ that must be visited on any tour visiting $v_{i}$. Now, for all $S \subset T \subseteq N \backslash\left\{v_{i}\right\}$, let $P_{S}^{*}\left(v_{0}\right)$ denote a minimum cost path from $v_{0}$ to a vertex in $Q \cup d(S)$. If $v_{0} \in Q \cup d(S)$, then the path is empty, and the cost is zero. Otherwise, it follows from the proof of case 1 that $\sum_{e \in P_{T}^{*}\left(v_{0}\right)} 2 t(e) \leq \sum_{e \in P_{S}^{*}\left(v_{0}\right)} 2 t(e)$. Next, consider $G \backslash E\left(G_{Q}\right)$, and let $G_{0}$ denote the connected component containing $v_{i}$ and $v_{0}$. Furthermore, let $\left(N^{\prime}, c_{v_{0}}^{\prime}\right)$ denote the 1-depot TS-game defined on $G_{0}$ in which $v_{0}$ is the single vertex associated with a depot, such that $N^{\prime}=V\left(G_{0}\right) \backslash\left\{v_{0}\right\}$, and $c^{\prime}$ is the restriction of $c$ to coalitions in $N^{\prime}$. Then, since the cut condition holds for all $v_{s}, v_{t} \in V\left(G_{0}\right)$, it follows from Herer and Penn [8] and Granot and Hamers [5] that $\left(N^{\prime}, c_{v_{0}}^{\prime}\right)$ is submodular, which in turn implies that

$$
\begin{aligned}
c_{Q}\left(T \cup\left\{v_{i}\right\}\right)-c_{Q}(T) & =c_{v_{0}}^{\prime}\left(\left\{T \cap V\left(G_{0}\right)\right\} \cup\left\{v_{i}\right\}\right)-c_{v_{0}}^{\prime}\left(\left\{T \cap V\left(G_{0}\right)\right\}\right)+\sum_{e \in P_{T}^{*}\left(v_{0}\right)} 2 t(e) \\
& \leq c_{v_{0}}^{\prime}\left(\left\{S \cap V\left(G_{0}\right)\right\} \cup\left\{v_{i}\right\}\right)-c_{v_{0}}^{\prime}\left(\left\{S \cap V\left(G_{0}\right)\right\}\right)+\sum_{e \in P_{S}^{*}\left(v_{0}\right)} 2 t(e) \\
& =c_{Q}\left(S \cup\left\{v_{i}\right\}\right)-c_{Q}(S),
\end{aligned}
$$

for all $v_{i} \in N \backslash V\left(G_{Q}\right)$ and all $S \subset T \subseteq N \backslash\left\{v_{i}\right\}$, where the inequality follows from the submodularity of $\left(N^{\prime}, c_{v_{0}}^{\prime}\right)$ and since $\sum_{e \in P_{T}^{*}\left(v_{0}\right)} 2 t(e) \leq \sum_{e \in P_{S}^{*}\left(v_{0}\right)} 2 t(e)$.

For the 'only if' part: First, if $G$ is not $P_{m+1}^{F}$-free with respect to $Q$ for $m \geq 4$, it follows from the proof of Theorem 4.1 that the induced game is not submodular for every weight function. Next, let $G_{j}$ be a connected component in $G \backslash E\left(G_{Q}\right)$, let $v_{j} \in V\left(G_{j}\right)$ be the unique vertex visited on any tour that visits $G_{j}$, and assume that there exists an $s-t$ vertex cutset of cardinality three or more for some $v_{s}, v_{t} \in G_{j}$. In Granot and Hamers [5], it was shown that a graph $G$ is locally 1-TS submodular if and only if the cut condition is satisfied for all vertex pairs $v_{s}, v_{t} \in V(G)$. It therefore follows that there exists no location of a single depot $v_{0}$ in $G_{j}$, such that an induced 1-depot TS game $\left(N^{\prime}, c_{v_{0}}^{\prime}\right)$ on $G_{j}$ is submodular for all weight functions. This in particular, is also the case for $v_{0}=v_{j}$, implying that $c_{v_{j}}^{\prime}\left(\left\{T \cap V\left(G_{j}\right)\right\} \cup\left\{v_{i}\right\}\right)-c_{v_{j}}^{\prime}\left(\left\{T \cap V\left(G_{j}\right)\right\}\right) \leq c_{v_{j}}^{\prime}\left(\left\{S \cap V\left(G_{j}\right)\right\} \cup\left\{v_{i}\right\}\right)-c_{v_{j}}^{\prime}\left(\left\{S \cap V\left(G_{j}\right)\right\}\right)$ does 
not hold for all $t$. Now, if we choose $S \subset T \subseteq N \backslash\left\{v_{i}\right\}$ such that $S \cap V\left(G_{j}\right) \neq \emptyset$, then $v_{j} \in d(S)$ (and hence, $v_{j} \in d(T)$ ), implying that $\sum_{e \in P_{S}^{*}\left(v_{j}\right)} 2 t(e)=\sum_{e \in P_{T}^{*}\left(v_{j}\right)} 2 t(e)=0$, since both paths are empty. It follows that (4.1) does not hold for all $t$, and $\left(N, c_{Q}\right)$ is not submodular for all weight functions. Therefore, there does not exist $Q$ with $|Q|=k$ such that every induced game on $G$ is submodular, and it follows that $G$ is not locally $k$-TS submodular.

\section{5. $k$-TS submodular directed graphs}

\subsection{Globally $k$-TS submodular digraphs}

Before we begin analyzing directed graphs, some notation is required. First, for any vertex pair $\left\{v_{i}, v_{j}\right\} \in V(G)$ such that a directed path from $v_{i}$ to $v_{j}$ exists, let $P_{i j}$ denote such a path. Second, let a 1-sum of two graphs $G_{1}$ and $G_{2}$ be the graph that is obtained by joining $G_{1}$ and $G_{2}$ by coalescing one vertex from $G_{1}$ with one vertex of $G_{2}$. The vertex joining the two former graphs will be referred to as the link vertex. Figure 6 illustrates the case where $G_{1}$ and $G_{2}$ are directed cycles of length 3.

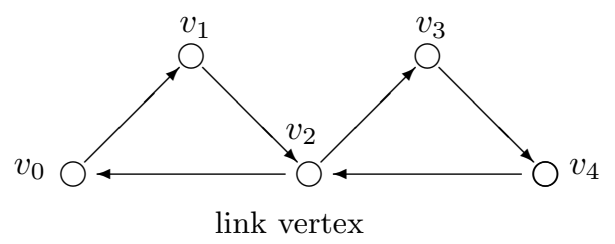

Figure 6: A 1-sum of two directed cycles

Next, note that a directed cycle $C$ with arc set $E(C)$ is globally $k$-TS submodular for all $k \in$ $\{2, \ldots,|V(G)|-3\}$, since $c(S)=\sum_{e \in E(C)} t(e)$ for all $S \subseteq N$. For digraphs in general, any induced game is submodular if $k \geq|V(G)|-2$, since the game is then either a one or a two-player subadditive game. Granot et al. 4 consider the case of $k=1$ and provide the equivalence theorem below. The graphs referred to as $F_{1}$ and $F_{2}$ are illustrated in Figure 7 .
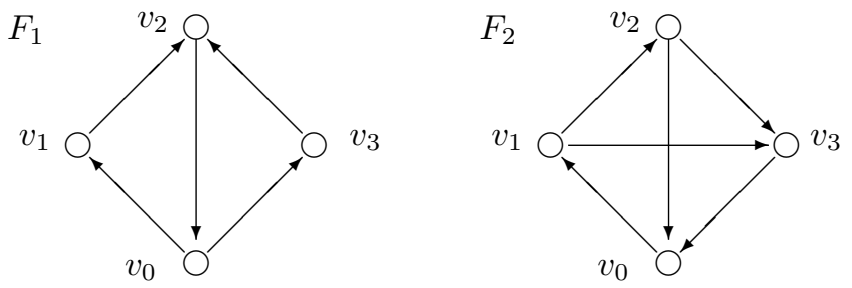

Figure 7: Forbidden subgraphs $F_{1}$ and $F_{2}$

Theorem 5.1. [Granot et al. [4]] Let $G$ be a strongly connected digraph. Then the following three statements are equivalent.

- $G$ is globally TS-submodular

- $G$ does not contain a subdivision of F1 or F2 
- $G$ is a 1-sum of harmonic digraphs each of which is an outerplanar graph with a directed cycle on its outer boundary $y^{2}$

If a directed graph $G$ contains a subdivision of $F_{1}$ or $F_{2}$, then for every 1-depot TSP on $G$ it is possible to choose a location of the single depot such that the induced game is not submodular for all weight functions $t$. Therefore, $G$ is not globally 1-TS submodular. A similar result holds for the case of $k>1$ as shown below.

Lemma 5.1. Let $G=(V(G), E(G))$ be a strongly connected, directed graph, and let $k \in\{2, \ldots,|V(G)|-$ $3\}$. If $G$ is globally $k$-TS submodular, then $G$ does not contain (a subdivision of ) $F_{1}$ or $F_{2}$.

Proof. Let $\Gamma=\left(V^{-}(G),(G, Q), t\right)$ be a multi-depot TSP on $G$. To arrive at a contradiction, assume first that $G$ contains (a subdivision of) $F_{1}$. Refer to $F_{1}$ in Figure 7 , and for all vertex pairs $v_{i}, v_{j} \in$ $V\left(F_{1}\right)$, let $\operatorname{cost}\left(P_{i j}\right)$ denote the cost of traversing the path $P_{i j}$ from $v_{i}$ to $v_{j}$. Let $t$ be a weight function on the arcs such that $\operatorname{cost}\left(P_{i j}\right)=1$ for all $P_{i j}$ such that $\left(v_{i}, v_{j}\right) \in E\left(F_{1}\right)$, and let $t(e)=100$ for all other $\operatorname{arcs}$ in $G$. Since we consider $k \leq|V(G)|-3$, we can choose $Q \subset V(G)$ such that $v_{0} \in Q$ and $v_{1}, v_{2}, v_{3} \in V^{-}(G)$. Next, let $S=\left\{v_{2}\right\}$ and $T=\left\{v_{1}, v_{2}\right\}$. Then $c_{Q}\left(T \cup\left\{v_{3}\right\}\right)-c_{Q}(T)=6-3=3>$ $0=3-3=c_{Q}\left(S \cup\left\{v_{3}\right\}\right)-c_{Q}(S)$, and the induced game is not submodular.

Assume instead that $G$ contains (a subdivision of) $F_{2}$. Refer to $F_{2}$ in Figure 7 and let $\operatorname{cost}\left(P_{i j}\right)=1$ for all $\left\{v_{i}, v_{j}\right\}$ such that $\left(v_{i}, v_{j}\right) \in E\left(F_{2}\right)$. Let $t(e)=100$ for all other $\operatorname{arcs}$ in $G$. Then if $S=\left\{v_{1}\right\}$ and $T=\left\{v_{1}, v_{2}\right\}$, we have $c_{Q}\left(T \cup\left\{v_{3}\right\}\right)-c_{Q}(T)=4-3=1>0=3-3=c_{Q}\left(S \cup\left\{v_{3}\right\}\right)-c_{Q}(S)$. Again, the induced game is not submodular, and therefore, $G$ is not globally $k$-TS submodular for any $k \in\{2, \ldots|V(G)|-3\}$.

As noted above, any directed cycle $C$ is globally $k$-TS submodular for all $k \in\{2,|V(C)|-3\}$, and it follows from Theorem 5.1 that 1 -sums of directed cycles are globally 1-TS submodular. However, 1sums of directed cycles or more generally, 1 -sums of directed circuits are not globally $k$-TS submodular for $k>1]^{3}$ In fact, if $G$ contains a 1-sum of directed cycles (or circuits) of at least three vertices each, then $G$ is not globally $k$-TS submodular for any $k \in\{2, \ldots,|V(G)|-3\}$.

Lemma 5.2. Let $G=(V(G), E(G))$ be a strongly connected, directed graph. Let $k \in\{2, \ldots,|V(G)|-$ $3\}$. If $G$ contains a 1 -sum of two directed circuits $C^{1}$ and $C^{2}$ such that each circuit is a closed walk of length three or more, then $G$ is not globally $k$-TS submodular.

Proof. Let $\Gamma=\left(V^{-}(G),(G, Q), t\right)$ be a multi-depot TSP on $G$, and let $\left(N, c_{Q}\right)$ be the induced $k$-depot TS game. To arrive at a contradiction, assume that a 1-sum exists as described in the statement and note that $\left|V\left(C^{1}\right)\right|,\left|V\left(C^{2}\right)\right| \geq 3$. Figure 6 illustrates the case of $G$ containing a 1-sum of two cycles with three vertices each, but the proof holds for cycles/circuits in general. Let $v_{2}$ denote the link vertex in this 1 -sum, and let $v_{0}, v_{1}$ be vertices in $C^{1}$ such that paths $P_{01}, P_{12}, P_{20}$ exist. Likewise, let $v_{3}, v_{4}$ be vertices in $C^{2}$ such that paths $P_{23}, P_{34}, P_{42}$ exist. Now, since the number of depots is $2 \leq k \leq|V(G)|-3$, we can choose $Q \subset V(G)$ such that $v_{1}, v_{2}, v_{3} \in V^{-}(G)$ and $v_{0}, v_{4} \in Q$, implying that there exists a depot in both $C_{1}$ and $C_{2}$ that is not the link vertex. Next, define the weight function $t$ on $G$ as follows: for any path $P_{i j} \in\left\{P_{01}, P_{12}, P_{20}, P_{23}, P_{34}, P_{42}\right\}$ let $\sum_{e \in E\left(P_{i j}\right)} t(e)=1$, and let $t(e)=100$ for all other $\operatorname{arcs}$ in $G$. Then, if $S=\left\{v_{2}\right\}$ and $T=\left\{v_{1}, v_{2}\right\}$, we see that $c\left(S \cup\left\{v_{3}\right\}\right)-c(S)=3-3=0$, while $c\left(T \cup\left\{v_{3}\right\}\right)-c(T) \geq 1$. Thus, the induced game is not submodular, and $G$ is not globally $k$-TS submodular.

Let $C_{2}$ denote a directed cycle with only 2 vertices. Then from Theorem 5.1 and Lemmas 5.1 and 5.2 we can infer the following:

\footnotetext{
${ }^{2}$ In Granot et al. 4, a digraph $G$ is said to be harmonic if every pair of cycles in $G$ visit their common vertices in the same order, i.e., all pairs of cycles are in harmony.

${ }^{3}$ Recall that a directed circuit is a closed walk in which no edge is visited more than once, while a directed cycle denotes a closed path, implying that neither vertices nor edges are visited more than once.
} 
Theorem 5.2. Let $G=(V(G), E(G))$ be a strongly connected, directed graph, and let $k \in\{2, \ldots,|V(G)|-$ $3\}$. If $G$ is globally $k$-TS submodular, then $G$ is a 1-sum of a harmonic, outerplanar graph, $C$, with a directed cycle on its outer boundary and (copies of) $C_{2}$, such that no two $C_{2}$ graphs share a link vertex in $V(G) \backslash V(C)$.

In the remainder of this section, we focus on the case where $G$ is an oriented graph, implying that $G$ contains no bi-directed edges, and hence no $C_{2}$. It is shown that even for graphs fulfilling the requirements of Theorem 5.2, the answer to whether the graph is globally $k$-TS submodular depends on the specific structure of the graph and the number of depots.

It follows from Granot et al. 4 that if $G$ is a harmonic and outerplanar graph with a directed cycle on its outer boundary, then this is sufficient for $G$ to be globally 1-TS submodular. Theorem 5.3 shows that the same does not hold for the case of $k \geq 2$.

Theorem 5.3. Let $G=(E(G), V(G))$ be a harmonic and outerplanar graph with a directed cycle on its outer boundary. Let $G$ contain at least one directed cycle $C_{i}$ such that $G \backslash V\left(C_{i}\right)$ is a collection of weakly connected components for which at least two distinct components contain 2 vertices or more. Let $C_{0}$ denote the $C_{i}$ with the minimum number of arcs. Then $G$ is globally $k$-TS submodular if and only if $k>|V(G)|-\left(\left|V\left(C_{0}\right)\right|+2\right)$.

Proof. Let $\Gamma=\left(V^{-}(G),(G, Q), t\right)$ be a multi depot TSP on $G$, and let $\left(N, c_{Q}\right)$ be the induced $k$-depot TS game. For the if part, it needs to be shown that 2.1) holds for all $v_{i} \in N$ and all $S \subset T \subseteq N \backslash\left\{v_{i}\right\}$. Note that if $S=\emptyset$, the result follows from the subadditivity of $c$, so we consider $S \neq \emptyset$. Furthermore, if $v_{i} \in d(T)$, then $c\left(T \cup v_{i}\right)-c(T)=0 \leq c\left(S \cup\left\{v_{i}\right\}\right)-c(S)$, and 2.1] holds. Therefore, it only remains to be shown that (2.1) holds when $v_{i} \notin d(T)$ and $S \neq \emptyset$.

Recall that $G$ is an outerplanar graph with a directed cycle on its outer boundary and denote this cycle by $C$. Let $v_{0} \in Q$ be a depot in $d(T)$ and number the vertices of $C$ in the order they are visited in a tour starting from $v_{0}$ and ending at $v_{|V(G)|}=v_{0}$. Consider a player vertex $v_{i} \notin d(T)$, and let $h=\max \left\{l \mid l<i, v_{l} \in d(T)\right\}$. Then there exists a vertex pair $v_{h}, v_{j} \in d(T)$ such that $j>i$, the arc $\left(v_{h}, v_{j}\right)$ - denoted $e_{h j}$ - exists, and $e_{h j} \in d(T)$. Furthermore, since all the vertices of $G$ lie on $C$, there also exists a path from $v_{h}$ to $v_{j}$ that visits $v_{i}$. Let $P_{h i j}$ denote the minimum cost path from $v_{h}$ to $v_{j}$ that visits $v_{i}$. Then since $d(T)$ is a minimum cost tour, we must have $t\left(e_{h j}\right) \leq \sum_{e \in P_{h i j}} t(e)$. Next, consider $S \subset T$ such that $S \neq \emptyset$, and let $g=\max \left\{l \mid l<i, v_{l} \in d(S)\right\}$. We consider two cases separately, depending on whether $v_{i}$ belongs to $d(S)$ or not.

Case 1. $v_{i} \notin d(S)$ : there exists a vertex $v_{k}$, such that $k>i$ and such that the arc $\left(v_{g}, v_{k}\right)$ - denoted $e_{g k}$ - exists and belongs to $d(S)$, and such that for a minimum cost path $P_{g i k}$ from $v_{g}$ to $v_{k}$ that visits $v_{i}$, it holds that $t\left(e_{g k}\right) \leq \sum_{e \in P_{g i k}} t(e)$. Furthermore, since $G$ is harmonic and outerplanar with a directed cycle on its outer boundary, it follows from Theorem 5.1 that $G$ does not contain the forbidden structure, $F_{2}$. This in turn implies that for the vertices $\left\{v_{h}, v_{j}, v_{g}, v_{k}\right\}$, we cannot have $g<h<i<k<j$, or $h<g<i<j<k$, and therefore, it must be that either $g \leq h<i<j \leq k$, or $h \leq g<i<k \leq j$ 迎 We consider the two cases separately:

Case 1.1. $h \leq g<i<k \leq j$. Note that since only the endpoints of $P_{h i j}$ are in $d(T)$, no vertices on $C$ that lie between $v_{h}$ and $v_{j}$ belong to $T$, implying that no vertices between $v_{h}$ and $v_{j}$ belong to $S$. Next, assume first that no depot exists on the part of $d(S)$ that lies between $v_{h}$ and $v_{j}$ (and goes via $e_{g k}$ ). Then we must have $t\left(e_{h j}\right)=\sum_{e \in P_{h i j}} t(e)-\sum_{e \in P_{g i k}} t(e)+t\left(e_{g k}\right)$ for both $d(S)$ and $d(T)$ to be minimum cost tours. This implies $c\left(T \cup\left\{v_{i}\right\}\right)-c(T)=\sum_{e \in P_{h i j}} t(e)-t\left(e_{h j}\right)=\sum_{e \in P_{g i k}} t(e)-t\left(e_{g k}\right)=c\left(S \cup\left\{v_{i}\right\}\right)-c(S)$, and (2.1) holds ${ }^{5}$ Now, let $C_{S \cup\{h, j\}}$ denote the smallest cycle that visits $S \cup\left\{v_{h}, v_{j}\right\}$. If $C_{S \cup\{h, j\}} \cap Q \neq \emptyset$, then the same reasoning as above applies. Therefore, consider the case of $C_{S \cup\{h, j\}} \cap Q=\emptyset$ and assume that there exists a depot on the part of $d(S)$ that lies between $v_{h}$ and $v_{j}$. Then since $G$ is outerplanar with a directed cycle on the outer boundary, we know that for $d(T)$ to be a minimum cost tour, there

\footnotetext{
${ }^{4}$ See Appendix A for illustrations of the two cases not considered.

${ }^{5}$ Note that in case $h=g, k=j$. We simply have $P_{h i j}=P_{g i k}$ and $e_{h j}=e_{g k}$.
} 
must exist a vertex pair $v_{l}, v_{m}$ on $C_{S \cup\{h, j\}}$ such that $v_{l}, v_{m}$ are the endpoints of a path $P_{l m}$ of which no other vertices belong to $S \cup\left\{v_{h}, v_{j}\right\}$, and such that at least one $v \in V\left(P_{l m}\right) \backslash\left\{v_{l}, v_{m}\right\}$ is a depot. First, in case $T \cap V\left(P_{l m}\right) \backslash\left\{v_{l}, v_{m}\right\}=\emptyset$, we must have $\sum_{e \in P_{l m}} t(e)=\sum_{e \in P_{h i j}} t(e)-\sum_{e \in P_{g i k}} t(e)+t\left(e_{g k}\right)$, and again, the desired result follows. Secondly, if $T \cap V\left(P_{l m}\right) \backslash\left\{v_{l}, v_{m}\right\} \neq \emptyset$, this implies that $G \backslash V\left(C_{S \cup\{h, j\}}\right)$ contains two weakly connected components with at least two vertices each, namely the paths $P_{h i j}$ and $P_{l m}$. This, however, implies that $k>|V(G)|-\left(\left|V\left(C_{h j}\right)\right|+2\right)$, which contradicts that $\left(C_{S \cup\{h, j\}}\right) \cap Q=\emptyset$. Case 1.2. $g \leq h<i<j \leq k$. Now, since $g \leq h$ and $j \leq k$, it must be that $\sum_{e \in P_{h i j}} t(e) \leq \sum_{e \in P_{g i k}} t(e)$. Next, since $v_{h}$ is the last vertex in $d(T)$ before $v_{i}$, and $P_{h i j}$ is the minimum weight path from $v_{h}$ to $v_{j}$, we have $c\left(T \cup v_{i}\right)-c(T)=\sum_{e \in P_{h i j}} t(e)-t\left(e_{h j}\right) \leq \sum_{e \in P_{g i k}} t(e)-t\left(e_{g k}\right)=c\left(S \cup\left\{v_{i}\right\}\right)-c(S)$, where the inequality follows since $t\left(e_{g k}\right) \leq \sum_{e \in P_{g i k}} t(e)-\sum_{e \in P_{h i j}} t(e)+t\left(e_{h j}\right)$ must hold for $d(S)$ to be a minimum cost tour. Thus, 2.1 holds.

Case 2. The case of $v_{i} \in d(S)$ remains to be considered. The reasoning is, however, similar to case 1 above. Like before, no vertex between $v_{h}$ and $v_{j}$ belongs to $S$, so if $V\left(P_{h i j}\right) \backslash\left\{v_{h}, v_{j}\right\} \cap Q=\emptyset$ and/or $C_{S \cup\left\{v_{h}, v_{j}\right\}} \cap Q \neq \emptyset$, we must have $t\left(e_{h j}\right)=\sum_{e \in P_{h i j}} t(e)$ for both $d(S)$ and $d(T)$ to be minimum cost tours. This implies $c\left(T \cup\left\{v_{i}\right\}\right)-c(T)=0=c\left(S \cup\left\{v_{i}\right\}\right)-c(S)$. Finally, if $C_{S \cup\left\{v_{h}, v_{j}\right\}} \cap Q=\emptyset$ and $V\left(P_{h i j}\right) \backslash\left\{v_{h}, v_{j}\right\} \cap Q \neq \emptyset$, we know that for $d(T)$ to be a minimum cost tour, there must exist a vertex pair $v_{l}, v_{m}$ on $C_{S \cup\{h, j\}}$ such that $v_{l}, v_{m}$ are the endpoints of a path $P_{l m}$ in $d(T)$ of which no other vertices belong to $S \cup\left\{v_{h}, v_{j}\right\}$, and such that at least one $v \in V\left(P_{l m}\right) \backslash\left\{v_{l}, v_{m}\right\}$ is a depot. Then either $P_{l m} \cap T=\emptyset$ implying that $\sum_{e \in P_{l m}} t(e)=\sum_{e \in P_{h i j}} t(e)$ and in turn that $c\left(T \cup\left\{v_{i}\right\}\right)-c(T)=0=c\left(S \cup\left\{v_{i}\right\}\right)-c(S)$, or $P_{l m} \cap T \neq \emptyset$. However, as before, the latter implies that $G \backslash V\left(C_{S \cup\{h, j\}}\right)$ contains two weakly connected components with at least 2 vertices each, contradicting that $C_{S \cup\left\{v_{h}, v_{j}\right\}} \cap Q=\emptyset$.

Next, consider the only if part and assume that $k \leq|V(G)|-\left(\left|V\left(C_{0}\right)\right|+2\right)$. Recall that $C_{0}$ is the shortest directed cycle in $G$ such that $G \backslash V\left(C_{0}\right)$ contains two weakly connected components with at least two vertices each, as illustrated in Figure 8. Let the two components be denoted $W_{1}$ and $W_{2}$ respectively. For each component $W$, let $P_{W}$ denote the shortest path between two vertices of $C_{0}$ that visits at least two vertices of $W$. Next, let $v_{0}$ be a vertex in $C_{0}$, let $v_{1}, v_{2}$ be vertices on $P_{W_{1}}$, and let $v_{3}, v_{4}$ be vertices on $P_{W_{2}}$.

Consider $\Gamma=\left(V^{-}(G),(G, Q), t\right)$, and note that for any $k \leq|V(G)|-\left(\left|V\left(C_{0}\right)\right|+2\right)$, we can choose $Q \subset V(G)$ such that $V\left(C_{0}\right) \subset V^{-}(G), v_{0}, v_{2}, v_{3} \in V^{-}(G)$, and $v_{1}, v_{4} \in Q$. That is, there are no depots located at any vertices of $C_{0}$, and (at least) one depot is located on each of $P_{W_{1}}$ and $P_{W_{2}}$. Next, define a weight function $t$ on $G$ as follows: for any $\left.e \in E\left(C_{0}\right) \cup E\left(P_{W_{1}}\right) \cup E\left(P_{W_{2}}\right)\right)$, let $t(e)=1$, and let $t(e)$ be arbitrarily high (e.g. $|E(G)|)$ for all other arcs in $G$. Without loss of generality, assume that $\left|E\left(P_{W_{2}}\right)\right| \geq\left|E\left(P_{W_{1}}\right)\right|$.

Let $S=\left\{v_{0}\right\}$. Then since there are no depots in $C_{0}$, a minimum cost tour of S visits $V\left(C_{0}\right) \cup V\left(W_{1}\right)$, and the cost of this tour is $c(S)=\left|E\left(C_{0}\right)\right|+\left|E\left(P_{W_{1}}\right)\right|-1$. It follows that $c\left(S \cup\left\{v_{2}\right\}\right)-c(S)=0$. Furthermore, let $T=\left\{v_{0}, v_{3}\right\}$. Then we have $c(T)=\left|E\left(C_{0}\right)\right|+\left|E\left(P_{W_{2}}\right)\right|-1$. However, since any tour of $T \cup\left\{v_{2}\right\}$ must visit both $V\left(P_{W_{1}}\right), V\left(P_{W_{2}}\right)$, and $V\left(C_{0}\right)$, it follows that $c\left(T \cup\left\{v_{2}\right\}\right)-c(T)=$ $\left|E\left(P_{W_{1}}\right)\right|-1>0$. Thus, the induced game is not submodular, and $G$ is therefore not globally $k$-TS submodular.

\subsection{Locally $k$-TS submodular digraphs}

Recall that for a graph $G$ to be locally $k$-TS submodular, we only require that one location of $k$ depots in $G$ induces a submodular game for all weight functions. Whereas all globally $k$-TS submodular graphs are obviously also locally $k$-TS submodular, it is easy to show that the opposite is not true, and hence that the class of globally $k$-TS submodular graphs is a proper subset of the class of locally $k$-TS submodular graphs. 


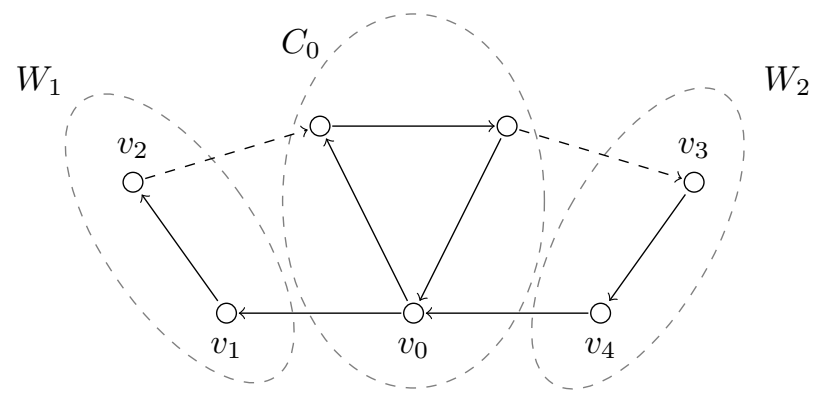

Figure 8: A globally 3-TS submodular graph that is not globally 2-TS submodular

Proposition 5.1. For $k \in\{2,|V(G)|-3\}$, the set of globally $k$-TS submodular graphs is a proper subset of the set of locally $k$-TS submodular graphs .

Proof. We only need to show that there exist graphs that are locally $k$-TS submodular but not globally $k$-TS submodular for $k \in\{2,|V(G)|-3\}$. To see this, consider the graph $G$ in Figure 9 . Since $G$ is a subdivision of $F_{1}$, it is not globally $k$-TS submodular, for $k \in\{1,2,3\}$. However, $G$ is locally $k$-TS submodular for $k=\{2,3\}$. For $k=2$, choose $Q=\left\{v_{0}, v_{1}\right\}$. It can readily be verified that for this location of depots, the induced game is submodular for any weight function. Thus $G$ is locally 2-TS submodular. Likewise, $G$ is locally 3-TS submodular, since the induced game is submodular whenever $Q$ is such that $\left\{v_{0}, v_{1}\right\} \in Q$. By further subdividing the graph in Figure 9 and increasing the number of depots correspondingly, the same argument can be applied for larger $k$.

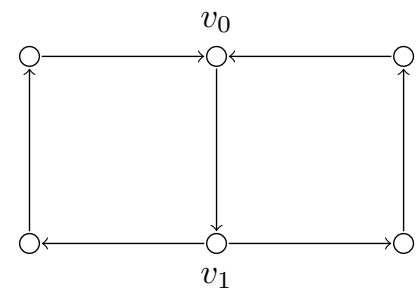

Figure 9: A locally (but not globally) 2-TS submodular graph

\section{Concluding remarks}

The current paper considers multi-depot traveling salesman games and analyzes the submodularity of such games by characterizing classes of graphs that induce submodular traveling salesman games. The main content of the results provided for undirected and directed graphs, respectively, is summarized in Table 1 .

The traveling salesman problem has been extended and varied in many directions, and for many applications the standard model is not sufficient. Therefore, one aim for future research on cost allocations problems arising from underlying traveling salesman problems is to allow for different (sets of) variations and/or restrictions in the underlying optimization problem, thereby making the model more easily applicable to real world situations. Examples include imposing capacity constraints or time windows for delivery. 


\begin{tabular}{c|l|l} 
& Globally $k$-TS submodular & Locally $k$-TS submodular \\
\hline $\begin{array}{c}\text { Undirected } \\
\text { graph }\end{array}$ & $\begin{array}{l}\text { If and only if } G \text { contains no path of } \\
\text { length four }\end{array}$ & $\begin{array}{l}\text { If and only if a) there exists a location } \\
\text { of } k \text { depots such that a forbidden struc- } \\
\text { ture is avoided, and b) the cut condition } \\
\text { holds for specific connected components }\end{array}$ \\
$\begin{array}{c}\text { Directed } \\
\text { graph }\end{array}$ & $\begin{array}{l}\text { Only if: a) } G \text { contains no forbidden sub- } \\
\text { graphs } F_{1} \text { and } F_{2}, \text { and b) } G \text { contains no } \\
\text { 1-sum of directed circuits each of length } \\
\text { three or more }\end{array}$ & $\begin{array}{l}\text { The set of globally } k \text {-TS submodular di- } \\
\text { graphs is a proper subset of the locally } \\
k \text {-TS submodular directed graphs }\end{array}$ \\
\hline
\end{tabular}

Table 1: Summary of results

\section{Aknowledgements}

Financial support from the Danish Council for Independent Research | Social Sciences (Grant IDs: DFF-1327-00097 and DFF - 6109-00132) is greatly acknowledged.

\section{Appendix A. Appendix}

To see that the remaining cases of $g<h<i<k<j$ and $h<g<i<j<k$ from the proof of Theorem 5.3 both imply that $G$ contains $F_{2}$, consider the illustrations in Figure A.1.
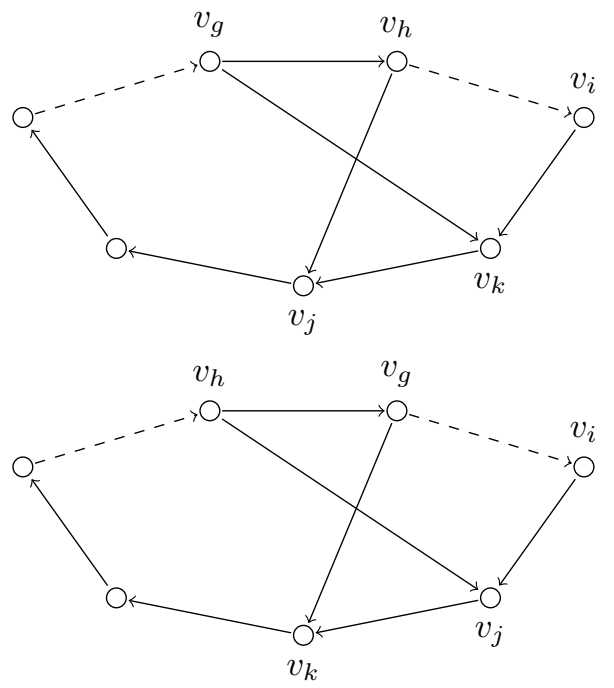

Figure A.1: $G$ with $g<h<i<k<j$ and $h<g<i<j<k$, respectively

\section{References}

[1] Curiel, I., 2010. Cooperative game theory and applications: cooperative games arising from combinatorial optimization problems. Kluwer Academic Publishers.

[2] Curiel, I., Potters, J., Prasad, R., Tijs, S., Veltman, B., 1994. Sequencing and cooperation. Operations Research 42 (3), 566-568. 
[3] Deng, X., Ibaraki, T., Nagamochi, H., Zang, W., 2000. Totally balanced combinatorial optimization games. Mathematical Programming 87, 441-452.

[4] Granot, D., Granot, F., Zhu, W. R., 2000. Naturally submodular digraphs and forbidden digraph configurations. Discrete Applied Mathematics 100(1-2), 67-84.

[5] Granot, D., Hamers, H., 2004. On the equivalence between some local and global chinese postman and traveling salesman graphs. Discrete Applied Mathematics 134(1-3), 67-76.

[6] Granot, D., Hamers, H., Tijs, S., 1999. On some balanced, totally balanced and submodular delivery games. Mathematical Programming 86(2), 355-366.

[7] Granot, D., Huberman, G., 1981. Minimum cost spanning tree games. Mathematical Programming $21(1), 1-18$.

[8] Herer, Y. T., Penn, M., 1995. Characterizations of natural submodular graphs: A polynomially solvable class of the TSP. Proceedings of the American Mathematical Society 123(3), pp. 673-679.

[9] Letchford, A. N., Nasiri, S. D., Theis, D. O., 2013. Compact formulations of the Steiner traveling salesman problem and related problems. European Journal of Operational Research 228 (1), 83 92.

[10] Maschler, M., Peleg, B., Shapley, L. S., 1971. The kernel and bargaining set for convex games. International Journal of Game Theory 1, 73-93.

[11] Platz, T. T., Hamers, H., 2015. On games arising from multi-depot chinese postman problems. Annals of Operations Research 235, 675-692.

[12] Potters, J. A. M., Curiel, I. J., Tijs, S. H., 1992. Traveling salesman games. Mathematical Programming 53 (1), 199-211.

[13] Shapley, L. S., 1971. Cores of convex games. International Journal of Game Theory 1(1), 11-26.

[14] Tamir, A., 1989. On the core of traveling salesman cost allocation game. Operations Research Letters 8, 31-34. 